\title{
Trivium
}

Revue franco-allemande de sciences humaines et sociales - Deutsch-französische Zeitschrift für Geistesund Sozialwissenschaften

$32 \mid 2021$

Institutions

\section{Matrix Reloaded}

Critique de l'approche stato-centrée de l'effet horizontal des droits fondamentaux à partir de l'exemple du biais de publication

\section{Isabell Hensel et Gunther Teubner}

Traducteur : Pierre Rusch

\section{(2) OpenEdition}

\section{Journals}

Édition électronique

URL : http://journals.openedition.org/trivium/7571

DOI : 10.4000 /trivium. 7571

ISBN : 1963-1820

ISSN : 1963-1820

\section{Éditeur}

Les éditions de la Maison des sciences de l'Homme

\section{Référence électronique}

Isabell Hensel et Gunther Teubner, "Matrix Reloaded », Trivium [En ligne], 32 | 2021, mis en ligne le 28 janvier 2021, consulté le 18 mars 2021. URL : http://journals.openedition.org/trivium/7571 ; DOI https://doi.org/10.4000/trivium.7571

Ce document a été généré automatiquement le 18 mars 2021.

\section{(c) (i) $\odot$}

Les contenus des la revue Trivium sont mis à disposition selon les termes de la Licence Creative Commons Attribution - Pas d'Utilisation Commerciale - Pas de Modification 4.0 International. 


\section{Matrix Reloaded}

Critique de l'approche stato-centrée de l'effet horizontal des droits fondamentaux à partir de l'exemple du biais de publication

Isabell Hensel et Gunther Teubner

Traduction : Pierre Rusch

\section{NOTE DE L'ÉDITEUR}

\section{Édition originale | Originalausgabe}

« Matrix Reloaded. Kritik der staatszentrierten Drittwirkung der Grundrechte am Beispiel des Publication Bias ", Kritische Justiz, 2014, p. 150-168.

Nous remercions Mme Isabell Hensel et M. Gunther Teubner de nous avoir accordé l'autorisation de traduire ce texte pour le présent numéro.

Wir danken Frau Isabell Hensel und Herrn Gunther Teubner für die freundliche Genehmigung, diesen Artikel in französischer Übersetzung zu publizieren.

À propos de l'expression « effet horizontal » :

Le terme Drittwirkung (mot-à-mot : effet sur les tiers) est utilisé en droit allemand pour désigner les conséquences normatives qu'ont les droits fondamentaux non pas seulement sur un plan vertical, pour l'État (contre les ingérences de qui ils ont été conçus) mais, horizontalement, pour les autres individus et groupes sociaux. D'où la traduction du terme par « effet horizontal ».

«Mais où sont les tableaux des noyés ${ }^{1}$ ?»

\section{Le biais de publication : la manipulation des études cliniques dans le réseau pharmaceutique}

1 L'affaire "Edronax»: l'antidépresseur Edronax des laboratoires Pfizer, utilisant la molécule réboxétine, fut autorisé en Allemagne et dans d'autres pays de l'Union européenne en 1997, alors qu'une demande d'autorisation était rejetée aux États-Unis. En 2010, le British Medical Journal révéla, ce que confirmèrent ensuite les travaux de 
l'IQWiG ${ }^{2}$, que Pfizer avait rendu public moins de deux tiers des études effectivement menées, ne retenant que celles qui aboutissaient à des résultats positifs. Le laboratoire avait en revanche passé sous silence celles qui prouvaient, par comparaison avec des placebos, que le médicament non seulement était inefficace, mais présentait aussi des effets secondaires indésirables.

2 L'affaire BASF contre Dong: Boots Pharmaceutical, qui s'appelle aujourd'hui Knoll Pharmaceutical Company, une filiale de BASF, chargea la scientifique Betty Dong de l'Université de Californie à San Francisco, contre un financement d'un quart de million de dollars, d'étudier l'efficacité du régulateur thyroïdien Synthroid, le médicament le plus prescrit aux États-Unis. En contrepartie, Dong devait s'engager à ne pas publier d'éventuels résultats négatifs sans l'accord du fabricant. De fait, le Synthroid ne présentait aucun avantage sur des médicaments génériques comparables et moins coûteux. En invoquant la clause contractuelle, en diffamant la chercheuse et ses méthodes, Boots empêcha pendant sept ans la publication de ses travaux. Le laboratoire put ainsi continuer à gagner des parts de marché en vantant la supériorité du Synthroid. Lorsque le Wall Street Journal porta l'affaire à la connaissance du public en 1996, BASF dut faire face devant les tribunaux à une plainte collective d'environ 5 millions d'utilisateurs pour rétention illégale de l'étude, publicité mensongère et atteinte aux règles de protection du consommateur. Le fabricant conclut finalement un arrangement avec les plaignants ${ }^{3}$.

3 L'affaire des "traitements hormonaux de substitution»: comme beaucoup d'autres laboratoires pharmaceutiques depuis les années 1940, Wyeth - qui s'appelle désormais Pfizer - organisa jusque dans les années 1990 des campagnes publicitaires sur les bienfaits des traitements hormonaux de substitution dans la prévention des troubles climactériques. Cette affirmation ne s'appuyait sur aucune étude scientifique solide. Il fallut attendre 1998 pour qu'une étude externe randomisée, suivie d'études complémentaires et d'une initiative de Women's Health en 2002, démontrent l'inexistence de tels effets préventifs, et mettent en évidence les risques de santé auxquelles s'exposaient les utilisatrices. Celles-ci, en effet, étaient plus souvent victimes de cancers du sein, d'AVC, de thromboses, de démence et d'incontinence. Des médias comme PLOS [Public Library of Science] et le New York Times obtinrent, parallèlement aux poursuites en dommages-intérêts des victimes, la divulgation de documents relatifs à la commercialisation du produit par le fabricant. Il apparut à cette occasion que les travaux sur lesquels s'appuyait la campagne publicitaire avaient été en grande partie conçus en collaboration avec des agences de communication et des prêteplumes ${ }^{4}$.

On peut dresser une longue liste de tels scandales, dans lesquels sont empêtrés les laboratoires pharmaceutiques. Il s'agit toujours d'empêcher ou de restreindre la diffusion publique de connaissances scientifiques concernant les effets néfastes ou l'inefficacité de telle ou telle substance médicamenteuse. Les modes de manipulation sont très divers, depuis les publications sélectives ${ }^{5}$ jusqu'aux clauses de censure dans les contrats de recherche, en passant par le recours à des prête-plumes, les pressions exercées sur les scientifiques ${ }^{6}$ pour les empêcher de mener leurs travaux, le licenciement de chercheurs par des institutions financièrement dépendantes des laboratoires ${ }^{7}$. Derrière ces affaires se dessine une collision de logiques d'action incompatibles, aboutissant à ce qu'on a appelé un «biais de publication ${ }^{8}$ ». Ce terme décrit une déformation statistique des données qui se produit lorsque les résultats des 
recherches sont étouffés ou manipulés dans certaines publications scientifiques. Il ne s'agit pas seulement d'incidents isolés regrettables, de scandales dans les pratiques scientifiques et sanitaires, qui, à ce titre, inquiètent l'opinion publique. De nombreuses études empiriques montrent que le biais de publication est un problème international, qui renvoie à des conflits d'intérêts massifs entre les institutions de recherche, l'industrie pharmaceutique, le système de santé, les maisons d'édition, les financeurs et les instances politiques de régulation. Une enquête comparant les protocoles et les publications ultérieures de 102 études de médicaments a ainsi établi que dans $62 \%$ des cas la publication s'écartait substantiellement du protocole d'évaluation ${ }^{9}$. On voit de plus en plus souvent des résultats négatifs, et donc indésirables, parce que défavorables à la commercialisation des produits étudiés, être bloqués ou manipulés, tandis que seuls les résultats positifs sont publiés dans les revues spécialisées. Ainsi, une partie seulement des études cliniques menées parvient au public. De telles suppressions répondent aux intérêts de l'industrie pharmaceutique, soucieuse que les études cliniques arrivent à des conclusions positives susceptibles de peser sur l'homologation et la commercialisation des produits. Elle cherche à obtenir satisfaction par le biais du financement des recherches, en intervenant d'une manière plus ou moins subtile dans le processus scientifique lui-même. De telles manipulations ne portent pas seulement préjudice à la science, elles nuisent aussi au système de santé.

5 Il ne suffit pas de décrire le biais de publication comme un effet de la corruption individuelle, auquel les instances étatiques de régulation pourraient apporter une réponse. $\mathrm{Du}$ fait des activités planétaires des groupes pharmaceutiques et de la mondialisation du travail scientifique, le conflit a pris des dimensions transnationales ${ }^{10}$. Il renvoie en même temps à un conflit structurel, qui peut être ponctuellement corrigé, mais pas efficacement maîtrisé par des mesures politiques. Derrière les affaires particulières se cache en effet un problème horizontal de droit fondamental : le conflit entre différentes rationalités sociales. L'atteinte à des domaines d'autonomie liés aux droits fondamentaux relève de la politique sous l'angle de la régulation, des tribunaux sous l'angle des arbitrages, mais surtout - d'une manière encore beaucoup plus décisive et avant toute considération de doctrine étatique quant aux droits fondamentaux - de l'initiative des acteurs sociaux sous l'angle de l'autorégulation. En ce sens, nous allons considérer les effets sociaux de ces pratiques sur les droits fondamentaux et leurs répercussions sur la politique et le droit.

\section{L'effet horizontal des droits fondamentaux : critique et alternatives}

6 Les droits fondamentaux comme outils de règlement des conflits permettent-ils de surmonter ce conflit d'intérêts à plusieurs dimensions, impliquant à la fois le niveau national et le niveau transnational? Manifestement, les intérêts des laboratoires pharmaceutiques transnationaux, soucieux de commercialiser avec succès leurs produits, entrent ici en conflit avec ceux de la communauté scientifique des chercheurs, qui veulent quant à eux pouvoir publier librement leurs résultats, ainsi qu'avec ceux des patients, attachés à une protection efficace de leur santé. Sur un plan juridique, cette situation s'inscrit dans le cadre de l'effet horizontal des droits fondamentaux, selon lequel des acteurs peuvent faire valoir leurs droits fondamentaux - en l'occurrence la liberté de la recherche scientifique et le droit à la santé - non 
seulement vis-à-vis des instances étatiques, mais aussi face à des acteurs privés ${ }^{11}$. Le concept d'effet horizontal implique un transfert des droits fondamentaux, du domaine du droit public à celui des relations de droit privé. Il importe ici de s'assurer que les principes porteurs du droit privé ne soient pas mis à mal dans cette opération. C'est la raison pour laquelle on rejette généralement l'idée d'un effet horizontal direct, et que l'on admet seulement un effet horizontal indirect, où l'ordre axiologique objectif des droits fondamentaux vient se couler dans les clauses générales et axiologiquement non qualifiées du droit privé, avant d'être adressé à l'instance judiciaire. Parallèlement à quoi, la théorie des obligations de protection introduit une responsabilité du législateur concernant le respect des droits fondamentaux dans les rapports de droit privé. En pratique, toutes les approches basées sur l'effet horizontal se ramènent à réaliser au cas par cas une mise en balance des droits fondamentaux contradictoires des sujets de droit privé.

Comparé à la conception traditionnelle des droits fondamentaux, qui se situait uniquement sur le plan de la relation individu-État, l'effet horizontal semble représenter un progrès significatif. Il répond à l'émergence de puissances sociales intermédiaires par le transfert de normes de droit public dans les relations de droit privé. Mais c'est justement dans cette image du transfert que réside le problème. Les différences entre le contexte de départ et le contexte d'arrivée sont généralement si grandes qu'elles interdisent un transfert de normes au sens strict. Il s'agit plutôt d'une reconstruction autonome des droits fondamentaux en fonction du contexte d'arrivée. La métaphore du transfert peut certes encore se lire comme une sorte de sémantique provisoire et intermédiaire, selon laquelle des droits fondamentaux dirigés contre l'État se trouvent "transposés" à des relations de droit privé et dotés d'un "effet horizontal» sur des acteurs sociaux. Mais à la longue, les droits fondamentaux intrasociaux ne peuvent être compris qu'à partir de leur contexte propre, qui est celui des conflits sociaux. Car les menaces potentielles qui pèsent ici sur les droits fondamentaux sont d'une tout autre nature que celles qui émanent de la sphère politique. Non moins différentes sont les situations d'atteinte aux droits fondamentaux et les sanctions appropriées dans chaque cas, de sorte que la simple idée d'un « effet horizontal » de droits fondamentaux initialement dirigés contre l'État est en soi une fausse piste.

$8 \quad$ Il s'agit donc de repenser l'effet horizontal des droits fondamentaux dans le droit privé en l'affranchissant de sa référence tacite à l'État, et d'en développer les normes directement à partir des particularités des conflits internes à la société. C'est pourquoi nous entreprendrons ici, sur l'exemple du biais de publication, de soumettre à un examen critique les quatre aspects centraux de la dogmatique de l'effet horizontal, et de proposer des alternatives.

9 Sous forme de thèses, on soutient ce qui suit :

1. La notion d'effet horizontal a jusqu'à présent été développée dans le sens d'une mise en balance des droits fondamentaux individuels. Or pour pouvoir surmonter les conflits structurels les plus graves au sein de la société, il est nécessaire, justement dans le secteur privé, de renforcer la dimension collective et institutionnelle des droits fondamentaux.

2. Au lieu de se limiter à la protection contre une puissance quasi-étatique au sein de la société, comme c'était le cas jusqu'à présent, la théorie de l'effet horizontal doit se dresser bien plus largement contre l'ensemble des médias de communication porteurs de tendances expansionnistes. 
3. La contextualisation des droits fondamentaux ne doit pas se borner à les adapter aux particularités du droit privé. Elle doit aller plus loin et tenir compte de la normativité propre des domaines d'autonomie menacés.

4. Au lieu d'imposer des obligations de protection exclusivement aux acteurs étatiques, la théorie de l'effet horizontal doit s'attaquer directement aux menaces sociales qui pèsent sur les droits fondamentaux et activer des contre-forces sociales.

\section{Les droits fondamentaux comme institution collective [Kollektiv- Institution]}

Une première critique vise la conception dominante de l'effet horizontal comme mise en balance des droits fondamentaux individuels ${ }^{12}$. Si l'on comprend l'effet horizontal comme transfert des droits fondamentaux reconnus par l'État vers la sphère des relations de droit privé, on perd de vue qu'un transfert pur et simple modifie la structure des droits fondamentaux et réduit la protection juridique. Au lieu qu'une atteinte aux droits fondamentaux soit examinée quant à son caractère illégal, des sujets de droit privé se trouvent identifiés comme auteur et victime d'un abus, et leurs situations respectives sur le plan des droits fondamentaux (qu'ils partagent au même titre) sont amenées à une "concordance pratique» dans tel cas particulier ${ }^{13}$. On n'obtiendra, de la sorte, rien de plus qu'un gain purement formel relativement à la protection des droits subjectifs dans le droit délictuel. Au contraire, la protection juridique se trouve en ce cas réduite, puisque les atteintes au droit fondamental sont beaucoup plus difficiles à établir, que les niveaux d'arbitrage se multiplient, et que les espaces politiques d'arbitrage s'étendent ${ }^{14}$. Le fait que la décision sur les atteintes à un droit fondamental soit rendue dépendante des circonstances particulières à chaque cas interdit en outre de formuler des normes générales pour des questions d'une telle portée. Cela conduit à une casuistique incontrôlable sur le plan conceptuel.

11 Mais l'objection la plus forte contre une approche exclusivement focalisée sur les droits individuels est qu'elle méconnaît la problématique centrale des atteintes intrasociales aux droits fondamentaux. Alors qu'il est admis depuis longtemps en droit public que les droits fondamentaux servent à protéger autant les droits individuels que les institutions sociales ${ }^{15}$, l'effet horizontal en droit privé, jusqu'ici, ne vise généralement que la protection des individus, en négligeant celle des institutions. La Cour constitutionnelle fédérale ne voit ici qu'un conflit entre les droits subjectifs individuels de "détenteurs de droits fondamentaux de même rang", entre des "droits fondamentaux concurrents ${ }^{16} »$. Et c'est sur ce plan des droits individuels que répondent les critiques provenant du droit privé1 ${ }^{17}$. Les uns et les autres perdent de vue que la dimension collective-institutionnelle des droits fondamentaux passe ici au premier plan.

Or c'est dans le conflit entre institutions sociales collectives que réside la problématique vraiment brûlante de l'effet horizontal des droits fondamentaux. Le concept de « collectif-institutionnel » indique qu'il est fait ici référence à la théorie des «droits fondamentaux impersonnels» de Helmut Ridder, selon laquelle «les droits fondamentaux visent la liberté concrète d'un champ social à travers son organisation ${ }^{18}$ ». Il faut notamment souligner que, au rebours des représentations traditionnelles, l'institution ne doit pas être comprise ici comme une garantie étatique de ce qui existe - selon la définition donnée par Carl Schmitt des garanties 
institutionnelles «se rapporte[nt] toujours à une réalité présente, à un existant formé et organisé, actuellement donné19 "-, mais comme un processus de normation juridique ouvert à des processus de normation sociale et en même temps soumis à des transformations incessantes.

Dans le cas du biais de publication, il serait certes parfaitement concevable que des scientifiques fassent valoir leurs droits individuels contre la censure des multinationales, qu'ils invoquent la nullité des contrats bloquant la publication de leurs travaux ou que des patients réclament des dommages et intérêts. Mais ce serait faire l'impasse sur la dimension collective-institutionnelle et, par là, sur les conflits sociaux les plus graves. Car les manipulations des laboratoires pharmaceutiques ne portent pas seulement atteinte aux droits individuels des chercheurs et des patients, ils violent aussi et plus fondamentalement l'intégrité et donc le fonctionnement des domaines sociaux autonomes que sont la science et le système de santée ${ }^{20}$.

Il faut en particulier souligner que la dimension collective-institutionnelle joue un rôle de part et d'autre de l'axe horizontal des droits fondamentaux. Si l'on trouve parmi les victimes non seulement des individus, mais aussi des institutions, il est également vrai que les responsabilités sont imputables, du côté des contrevenants, autant à des processus sociaux anonymes qu'à des personnes. Ce caractère bilatéral de la relation collective-institutionnelle est souvent perdu de vue. Mais la discussion des pénalistes sur la macrocriminalité et la responsabilité pénale des organisations formelles, qui s'adosse au débat sociologique sur la violence structurelle, a déjà développé une telle approche collective-institutionnelle du côté du contrevenant; il conviendra d'en tirer parti quant à l'effet horizontal des droits fondamentaux ${ }^{21}$.

Les atteintes aux droits fondamentaux, dans de tels cas, sont à rapporter à des processus sociaux impersonnels, qui se servent d'acteurs humains comme de simples exécutants ${ }^{22}$. La violence structurelle procède de la "matrice anonyme ", c'est-à-dire non seulement d' "acteurs collectifs" assez identifiables (États, partis politiques, entreprises, trusts, consortiums), mais aussi - avec une intensité comparable, voire supérieure - de processus communicationnels autonomisés (institutions, systèmes fonctionnels, réseaux) qui ne sont justement pas identifiés comme des acteurs collectifs $^{23}$. Les menaces qui émanent des processus numériques sur Internet illustrent parfaitement cette situation ${ }^{24}$. Au centre du conflit se trouve la collision de logiques d'action inconciliables: l'agir économiquement rationnel corrompt structurellement les logiques propres de la science et du système de santé. Et la particularité de cette collision réside dans son asymétrie. La protection des droits fondamentaux est nécessaire dans de telles situations d'asymétries, où l'envahissante dynamique économique neutralise les fragiles mécanismes internes de la science et du système de santé.

Envisager les droits fondamentaux comme des institutions collectives signifie donc instaurer une relation bilatérale, dans laquelle l'autonomie de certains processus sociaux est garantie contre les tendances totalisantes d'autres processus sociaux ${ }^{25}$. Dans cette dimension collectiveinstitutionnelle, les droits fondamentaux fonctionnent comme des outils de règlement des conflits, qui interviennent lorsque des rationalités sociales partielles entrent en contradiction entre elles. Ils visent à protéger l'intégrité de l'art, de la famille, de la religion, contre des tendances totalisatrices existant dans la société, comme celles de la technologie, des médias ou de l'économie. Il serait manifestement inutile, en ce domaine, d'essayer de mettre en balance des droits fondamentaux individuels. 

des droits fondamentaux sur le terrain de l'organisation et de la procédure. Des mesures visant à la protection institutionnelle des espaces sociaux d'autonomie ont depuis longtemps été introduites dans le droit public, en particulier dans le droit des médias $^{26}$. Dans le contexte des médias de masse, la liberté d'opinion ne peut être efficacement protégée par les droits subjectifs, il faut en passer par l'organisation et la procédure ${ }^{27}$. Ce constat doit être généralisé et appliqué précisément à l'effet horizontal des droits fondamentaux dans différents domaines sociaux. collective-institutionnelle des droits fondamentaux se trouve en adéquation avec le contexte. L'organisation et la procédure doivent être choisies de manière à prendre en compte les contextes spécifiques de part et d'autre de l'atteinte aux droits fondamentaux - du côté de la victime comme du côté du contrevenant ${ }^{28}$. Dans le cas du biais de publication, la question centrale est alors : à quelles conditions l'exploitation économique des résultats de la recherche scientifique devient-elle intrusive au point de violer l'intégrité essentielle de la recherche d'une part, du système de santé d'autre part? La recherche des critères doit donc s'effectuer dans deux directions différentes. Il faut se demander: $1^{\circ}$ À quelle menace spécifique les droits fondamentaux se trouvent-ils exposés, lorsque la publication de résultats scientifiques subit des pressions motivées par des considérations économiques? $2^{\circ}$ Comment, dans ces conditions, définir le noyau central de la science et du système de santé auquel la manipulation des résultats de la recherche a porté atteinte? C'est seulement lorsque nous aurons répondu d'une manière suffisamment précise à ces deux questions qu'il sera possible de dire comment l'organisation et la procédure doivent être conçues pour pouvoir restaurer l'intégrité violée de la science et du système de santé.

\section{La dynamique expansionniste des médias de communication}

19 Un deuxième point faible des théories traditionnelles de l'effet horizontal est qu'elles se concentrent exclusivement sur la protection contre le pouvoir social ${ }^{29}$. C'est ce que met particulièrement en lumière la doctrine de la state-action, dans la dogmatique étatsunienne des droits fondamentaux ${ }^{30}$. Un effet horizontal des droits fondamentaux est ici admis par analogie avec l'effet des droits fondamentaux vis-à-vis de l'État, dans le cas où certains acteurs privés jouissent d'un pouvoir socio-économique équivalent au pouvoir étatique ${ }^{31}$. La théorie directe et la théorie indirecte de l'effet horizontal font elles aussi référence à des situations structurelles de déséquilibre et de mise en danger, et ne prennent en compte que des phénomènes liés à l'exercice d'un pouvoir social.

Il est indiscutable que la protection juridique contre le pouvoir social constitue un important terrain d'application de l'effet horizontal, mais la faiblesse du schéma du transfert s'atteste ici aussi. Car limiter la défense des droits fondamentaux aux cas où s'est constituée au sein de la société une puissance privée d'une intensité comparable à la puissance étatique ne serait plausible que s'il s'agissait de transférer à des conflits intrasociaux des droits fondamentaux définis d'abord contre l'état. C'est pourquoi la théorie de l'effet horizontal a été si fructueuse dans le droit du travail, la propriété privée se trouvant transformée ici en pouvoir organisationnel du private government, dont les effets ne le cèdent en rien à ceux de l'exercice du pouvoir étatique ${ }^{32}$. 
21 Mais se focaliser sur le pouvoir social, c'est passer à côté d'autres causes, plus subtiles, à l'origine des violations collectives-institutionnelles des droits fondamentaux. Autant il est approprié que ceux-ci soient dirigés contre les manifestations de pouvoir dans le domaine étatique, autant il est contestable de les limiter à ce médium communicationnel qu'est le pouvoir, lorsque ce sont des forces sociales qui sont en cause. Les droits fondamentaux sont essentiellement menacés par tous les médias de communication autonomisés, dès lors que tel ou tel sous-système développe des dynamiques d'expansion propres. Il s'agit aujourd'hui avant tout des tendances expansionnistes de l'économie, de la technologie, de la médecine et, tout particulièrement, des médias d'information. Le pouvoir social ne représente donc qu'une partie des menaces qui pèsent sur les droits fondamentaux au sein de la société. Les différences essentielles entre les droits sociaux fondamentaux et les droits politiques fondamentaux résultent toujours des différentes conditions internes de reproduction des domaines concernés. Dans le domaine politique, les droits fondamentaux sont avant tout dirigés contre les dangers du pouvoir; dans d'autres systèmes sociaux, ils répondent aux menaces provenant de leurs médias communicationnels spécifiques - dans l'économie, des transactions financières; dans la science et la technologie, des opérations cognitives-techniques; dans les médias, des flux d'information ${ }^{33}$.

22 S'agissant du biais de publication, le pouvoir économique joue un rôle de premier plan. Les clauses de censure que l'industrie pharmaceutique impose aux chercheurs, en particulier, témoignent d'un rapport de forces asymétrique dans les relations contractuelles. Mais l'on ne doit pas se focaliser sur les phénomènes de pouvoir, il faut également se prémunir contre les prises d'influence plus subtiles qui, sans que le pouvoir se manifeste explicitement, "substituent à la pertinence scientifique des valeurs et des normes extra-scientifiques ${ }^{34} »$. Il faut notamment tenir compte de l'influence corruptrice des flux financiers, tout particulièrement lorsqu'ils ne se traduisent pas sur le plan du pouvoir organisationnel ou contractuel. La «technique de pouvoir » des laboratoires pharmaceutiques «n'est pas prohibitive ou répressive, elle est séductive [...] Elle séduit au lieu d'interdire ${ }^{35}$ ». Leur force de motivation ne repose pas sur le pouvoir exercé par des sanctions négatives, mais sur l'énorme besoin de financement de la recherche scientifique, auquel s'adressent précisément les techniques de séduction des laboratoires pharmaceutiques. «Parmi les conditions d'une recherche exigeante en personnel et en ressources, le financement de la science constitue le point névralgique de sa liberté ${ }^{36}$. " C'est aussi là l'une des raisons pour lesquelles les droits fondamentaux, quand ils sont conçus comme de simples défenses contre le pouvoir, restent désarmés face aux influences exercées par le moyen de l'argent. Une théorie de l'effet horizontal aura donc pour tâche de protéger efficacement les droits fondamentaux contre ces techniques de séduction ${ }^{37}$.

23 Toute prise d'influence économique sur la recherche scientifique ne constitue certes pas pour autant une atteinte aux droits fondamentaux. Il existe toute sorte de contacts entre l'économie et la science, parmi lesquels l'exploitation économique des résultats scientifiques, l'orientation des choix des thèmes de recherche (par exemple par la participation des entreprises aux instances des universités), le financement des projets les plus profitables, la pratique de la recherche industrielle, la recherche appliquée en général ou encore l'étroite coopération des entreprises et des laboratoires de recherche dans des contextes du type Silicon Valley ${ }^{38}$. Toutes ces pratiques peuvent provoquer des 
réglementations politiques, mais elles ne constituent pas une atteinte aux droits fondamentaux tant que l'autonomie de la science n'est pas mise en cause en son principe.

C'est seulement quand les influences extérieures manipulent systématiquement le code scientifique lui-même, autrement dit qu'elles cherchent à déterminer du dehors ce qui est vrai et ce qui est faux - comme ce fut le cas, par rapport au pouvoir politique, avec la biologie lyssenkiste en Union Soviétique -, c'est à ce moment-là seulement que le noyau dur du système scientifique est touché ${ }^{39}$. L'atteinte à liberté de la science est donc patente quand la rationalité économique usurpe l'autorité de la rationalité scientifique, quand elle remplace le code scientifique par le code économique. Or rien de tout cela n'a généralement lieu dans le cas du biais de publication. L'industrie pharmaceutique se garde bien d'intervenir elle-même directement dans le travail de recherche, ou de prescrire aux scientifiques les résultats auxquels ils doivent parvenir ${ }^{40}$. Une intervention externe aussi maladroite dans le code binaire de la science ou dans ses programmes ne ferait - comme l'a montré la désastreuse affaire Lyssenko que s'exposer au ridicule, face à une pratique scientifique stabilisée. Les manipulations économiques dont il est question ici sont beaucoup plus subtiles, et d'autant plus dangereuses, car elles s'insinuent imperceptiblement dans le processus scientifique, générant ainsi le biais de publication ${ }^{41}$. C'est la raison pour laquelle il est si compliqué d'en établir la réalité. Ce n'est qu'au terme de coûteuses recherches empiricostatistiques qu'il a été possible de prouver, comme nous l'avons décrit au début de cet article, que le processus de publication avait été systématiquement faussé.

Il est d'autant plus difficile, dans de telles configurations, de déterminer exactement de quelle manière l'autonomie de la science se trouve menacée. La thèse défendue ici est la suivante: Si les manipulations à l'origine du biais de publication touchent au noyau dur de la science, ce n'est pas parce qu'elles contreviennent directement à son code de vérité ou à ses programmes, à ses méthodes et à ses théories. Elles entravent plutôt l'évolution de la science parce qu'elles faussent systématiquement ce fragile mécanisme de sélection qu'est la publication. Les liens subtils entre la variation, la sélection et la rétention dans l'évolution de la science se trouvent alors soumis aux manipulations motivées par des considérations économiques, qui viennent brouiller le mécanisme de publication. Il en résulte des conséquences dramatiques pour le développement autonome du système scientifique (cf. infra, 1); en même temps, l'infiltration de la science par l'économie porte atteinte, dans l'interaction des espaces autonomes dont se compose la société, à l'intégrité du système de santé (cf. infra, 2).

\section{(1) Une atteinte au mécanisme de publication}

La publication représente dans l'évolution du système scientifique un mécanisme de sélection $^{42}$ qui, parmi les multiples variations des recherches en cours, retient les résultats pertinents pour le système. La publication originale dans les principales revues spécialisées a pour fonction de filtrer dans le champ les résultats qui vont déterminer l'orientation de l'évolution ultérieure. En rendant la nouveauté visible, elle opère une sélection parmi les diverses modifications subies par le processus scientifique à travers la mise en œuvre du code binaire ("vrai/faux») et des programmes; elle favorise ainsi la stabilisation des résultats de la recherche sous la forme d'un «état de l'art» exposé dans les manuels, lequel suscite à son tour de nouvelles variations ${ }^{43}$. La pratique de la publication amène de l'objectivité et de 
l'impartialité dans la science, car elle permet l'autocontrôle des connaissances scientifiques sur des critères d'articulation interne et d'exposition à la critique ${ }^{44}$. L'institution sociale d'une pratique publicationnelle efficace fait donc partie du domaine protégé de la liberté de la science, au même titre que la liberté de publication elle-même. On mesure ici l'interaction entre le niveau individuel et le niveau collectifinstitutionnel des droits fondamentaux. Ceux-ci se rapportent non seulement aux individus mais aussi «à des institutions collectives [...] qui ne peuvent être opposées au sujet, parce qu'elles participent aux processus de (re)production du sujet, sans pour autant constituer un macro-sujet ${ }^{45} »$. Les droits fondamentaux individuels ne sont pas limités par les institutions collectives, ils constituent l'espace où celles-ci se réalisent ${ }^{46}$. Inversement, les droits fondamentaux opposables des individus permettent aussi de plaider pour la protection et le développement des institutions collectives.

Les manipulations à finalité économique obèrent ce mécanisme, à la fois directement et indirectement. Directement: si les réseaux pharmaceutiques ne mobilisent pas leurs droits contractuels d'utilisation et d'exploitation, ou encore d'éventuelles clauses de censure, pour intervenir dans la « production » des résultats scientifiques, ils n'hésitent pas à le faire pour orienter leur " présentation ${ }^{47} »$. Des études négatives se trouveront ainsi mises sous le boisseau, leurs résultats manipulés, de sorte que la masse des publications penche vers des évaluations plus profitables, et que la répartition des résultats positifs et des résultats négatifs de la recherche évolue donc significativement en faveur des premiers.

Une atteinte indirecte se produit, à l'inverse, lorsque le besoin de financement prévaut sur le désir scientifique de connaissance. La publication de résultats positifs est alors plus lucrative et plus intéressante pour le chercheur que la publication de résultats négatifs ${ }^{48}$. La norme intrascientifique des "bonnes pratiques scientifiques", selon laquelle une telle publication sélective aurait constitué une faute, perd en pertinence ${ }^{49}$. Petit à petit, c'est la perception même de l'acte de publication qui se transforme au sein de la recherche scientifique. Un fait symptomatique de cette évolution est le recours de plus en plus fréquent et opaque à des agences dites de communication et à des prêteplumes. Des chercheurs très en vue, soucieux d'étendre leur réputation, assument la paternité d'études qu'ils n'ont pas menées, et qui ont été en réalité rédigées par des prête-plumes, des sociétés de conseil ou des collaborateurs des laboratoires pharmaceutiques ${ }^{50}$.

Certains éditeurs encouragent aussi de telles manipulations, lorsqu'ils adaptent leur pratique éditoriale aux attentes et aux pressions financières des laboratoires pharmaceutiques et acceptent prioritairement les études positives ${ }^{51}$. Il n'est pas rare que de grands éditeurs médicaux s'entendent avec les laboratoires pharmaceutiques, qui les financent par le biais de la publicité. Ces accords portent aussi bien sur l'orientation des revues spécialisées que sur les critères de publication ${ }^{52}$. À quoi s'ajoute dans le domaine pharmaceutique la difficulté de trouver pour le processus de peerreviewing des experts indépendants, de manière à éviter des conflits d'intérêts susceptibles de peser sur les résultats.

Quand les intérêts économiques influencent de la sorte les pratiques de publication, les critères de sélection internes à la science sont remplacés par des critères de sélection externes. Les procédures de peer-reviewing tournent à vide, parce que les données négatives n'apparaissent pas. Il n'est plus possible de s'appuyer sur ces travaux pour développer d'autres études dans le prolongement ou en parallèle des précédents; pire 
encore, la falsification se répercute dans les études ultérieures ${ }^{53}$. Car l'utilisation de données fausses dans de nouvelles recherches, finit par compromettre l'ordre de valeurs du code de vérité de la science elle-même. Les répercussions du biais de publication sur la pratique de la recherche tendent à dissoudre le lien entre les deux activités. L'autoreproduction de la recherche scientifique est menacée en son cœur.

\section{(2) Une atteinte au droit à la santé}

31 Une telle pratique porte atteinte au droit à la santé, à la fois sur le plan collectifinstitutionnel et sur le plan individuel. Les institutions collectives de la sphère politique et du système de santé, mais aussi les médecins traitants, doivent pouvoir avoir un accès illimité à toutes les études concernant tel ou tel médicament. Si des connaissances relatives aux effets nocifs d'une substance sont mises sous le boisseau ou manipulées, les acteurs n'auront pas une image objective de l'impact de son utilisation en raison de la sélection des données publiées par les revues spécialisées. Il en résultera des erreurs graves dans les choix effectués, puisque les effets positifs auront été surestimés tant au niveau de la procédure d'autorisation du médicament, que dans le traitement des patients ${ }^{54}$. Dans la mesure où les études cliniques (selon les $\S \S 21 \mathrm{sq}$. de la loi allemande sur les médicaments [Arzneimittelgesetz, AMG]) servent de fondement à l'autorisation d'exploitation, sans que les autorités sanitaires ne procèdent ensuite à leurs propres contrôles, de telles manipulations entraînent immédiatement une appréciation erronée de l'efficacité et de l'utilité des médicaments. Il en résulte que de fausses informations quant à l'efficacité figurent sur les emballages et que les caisses d'assurance maladie prennent des décisions inadaptées dans le remboursement des soins, comme le montre l'affaire Edronax. Les associations de médecins sont ainsi amenées à fournir des indications de traitement erronées. Des organes institutionnels de contrôle tels que le IQWiG [Institut für Qualität und Wirtschaftlichkeit im Gesundheitswesen, Agence fédérale du médicament] ou les comités d'éthique ne peuvent remplir leur rôle, car ils sont tributaires de données lacunaires ${ }^{55}$.

Les dangers pour les patients et les sujets d'expérience sont évidents. En raison de la falsification des études, des patients sont amenés à subir des traitements inutiles, voire dangereux. Des produits réellement efficaces demeurent ignorés, et ne sont pas mis à la disposition du public. La dissimulation d'études déjà réalisées conduit à soumettre inutilement des sujets d'expérience à de nouveaux tests ${ }^{56}$.

\section{Contextualisation}

33 Le troisième point faible des théories traditionnelles de l'effet horizontal tient à la conception erronée du transfert. D'ordinaire, l'effet horizontal est compris comme la transposition aux relations de droit privé de droits fondamentaux élaborés dans le domaine du droit public. Mais une telle transposition doit s'opérer, selon cette approche, dans le respect de certains principes fondateurs du droit privé. À cet égard, la théorie de l'effet horizontal indirect considère que l'adaptation au droit privé est assurée lorsque les droits fondamentaux s'introduisent dans le droit privé par le biais de clauses générales. La théorie des obligations de protection fait, elle, intervenir le législateur, qui pour les mêmes raisons ne doit formuler que des normes conformes au droit privé. 

fondamentaux régissant les rapports sociétaux. Mais c'est concevoir le nouveau contexte d'une manière beaucoup trop étroite que de l'assimiler au monde du droit privé. L'exigence de maintenir, au cours de ce transfert, "l'indépendance et l'autonomie fondamentale du droit civil face au système des droits fondamentaux tel qu'énoncé en droit constitutionnel ${ }^{57} \gg$ ne décrit qu'un premier pas dans le processus de contextualisation. Le deuxième pas nous confronte à une tâche autrement plus difficile : les droits fondamentaux originellement dirigés contre l'État ne doivent pas être adaptés seulement au contexte du droit privé, mais aussi aux différents contextes sociaux. Ils doivent être ajustés de façon à protéger la logique et la normativité propre des différents domaines sociaux partiels exposés à ce genre de violations des droits fondamentaux ${ }^{58}$.

C'est ici, à tout le moins, que l'idée du transfert atteint ses limites. Si, dans un cas particulier à caractère individuel, les droits fondamentaux élaborés sur le plan du droit public peuvent encore être transposés avec quelque chance de succès dans la sphère des rapports de droit privé, le transfert institutionnel de droits fondamentaux, c'est-àdire le transfert d'un mode d'organisation défini au préalable et de procédures préétablies, ne peut qu'échouer, en raison de la multiplicité et de la spécificité des normativités sociales ${ }^{59}$. Une protection adéquate des droits fondamentaux ne peut être assurée au moyen d'une conception unique de la protection de ces droits appliquée à tous les secteurs sociaux ${ }^{60}$, mais seulement "sur le terrain", grâce à une contextualisation minutieuse et nuancée.

À la question de savoir quel mode d'organisation et quelles procédures servent à protéger sur le plan des droits fondamentaux les institutions collectives que sont la science et le système sanitaire contre les empiètements de l'économie, il faut répondre avant tout à partir de l'autocompréhension normative des pratiques sociales menacées ${ }^{61}$. La science et le système de santé élaborent dans leurs propres codes et leurs propres programmes des orientations normatives, qui ne se confondent pas avec les opinions conventionnelles des individus, et présentent au contraire un caractère

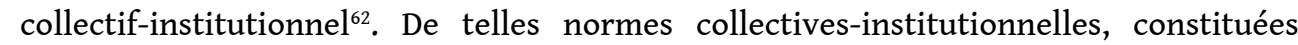
historiquement, sont soumises à la discussion et à la critique dans les discours réflexifs de la science et du système de santé, avant d'être examinées par le droit selon ses propres critères et établies en tant que normes juridiques. Concernant les manipulations à l'origine d'un biais de publication, les discours réflexifs de la science et du système de santé ont de fait développé une alternative collective-institutionnelle à la protection individuelle, dont il faut souhaiter qu'elle fasse l'objet d'une élaboration juridique $^{63}$ : l'enregistrement des essais [trial-registration] comme effet horizontal, par l'organisation et la procédure, de la liberté de la science et du droit à la santé64. Il s'agit d'obliger les chercheurs à tenir des registres accessibles au public, où ils doivent consigner dès le début de chaque étude leurs tests et leurs résultats, afin d'assurer la transparence et le contrôle de l'ensemble du processus de recherche ${ }^{65}$. Cette forme de protection des droits fondamentaux n'est efficace que si les revues spécialisées y coopèrent en conditionnant la publication des études à leur inscription préalable dans un tel registre ${ }^{66}$. Les résultats concernant des médicaments destinés à être mis sur le marché ne peuvent alors être publiés que lorsque les essais cliniques sur lesquels ils se fondent ont d'abord été inscrits au registre correspondant, avec tous leurs résultats positifs et négatifs ${ }^{67}$. La trial-registration vise tout particulièrement le conflit entre la 
rationalité économique et la logique propre de la science. Car dans la procédure et l'organisation de la protection des droits fondamentaux, l'obligation d'enregistrement porte précisément sur le point où, comme décrit plus haut, les manipulations faussent le processus d'évolution de la science. À la différence d'autres sanctions possibles, l'obligation d'enregistrement vise le mécanisme critique de sélection au sein duquel le discours de l'économie entre en collision avec celui de la science et de la santé. La trialregistration ne permet pas de contrecarrer les techniques de pouvoir répressives ou prohibitives des laboratoires pharmaceutiques, mais elle apporte un correctif à leurs techniques " séductives » de manipulation ${ }^{68}$. Elle génère de la transparence, mais, d'une manière plus décisive encore, elle stabilise et protège l'acte même de publication, en ne le limitant plus aux résultats finaux, et en l'élargissant à l'ensemble du projet de recherche. Et ce, avant même que ses résultats soient connus. Elle oblige ainsi les personnes impliquées à déterminer leur stratégie de publication sous un "voile d'ignorance ». Les projets doivent être rendus accessibles à la communauté médicale à un moment où les résultats de la recherche sont encore inconnus. La contingence du projet de recherche concret est ainsi rendue publique. Et la pratique de publication se trouve exposée à une pression systématique concernant la fréquence respective des résultats positifs et négatifs.

L'obligation d'enregistrement porte donc exactement sur le mécanisme de sélection de l'évolution scientifique, lequel ne fonctionne pas seulement au cas par cas, comme le font les plaintes individuelles, mais influence sur le long terme la co-évolution de l'économie, de la science et de la pratique médicale. Elle renforce le critère proprement scientifique de sélection des nouveaux développements, sans préjuger du caractère positif ou négatif des tests, et affaiblit les critères économiques qui génèrent le biais de publication. Et elle renforce simultanément les critères de sélection de la pratique médicale, pour laquelle la connaissance des effets secondaires indésirables ou de l'inefficacité des médicaments est aussi importante que l'information concernant leurs effets thérapeutiques positifs. Une telle obligation d'enregistrement tend à restaurer le lien entre la recherche et la publication, dont dépend essentiellement l'autoreproduction de la science et que visent à saboter les techniques séductives de manipulation mises en œuvre par les entreprises pharmaceutiques.

\section{Au-delà de l'obligation de protection : les alternatives à la réglementation étatique des pratiques de publication}

Même la conception aujourd'hui admise de l'obligation de protection, telle qu'elle a été développée dans le cadre de la théorie de l'effet horizontal, s'expose au reproche d'une focalisation trompeuse sur l'État : bien que ce soient des acteurs privés qui enfreignent les droits fondamentaux, l'obligation concerne prioritairement l'État et non ces acteurs eux-mêmes. Ce qui est particulièrement fâcheux dans le domaine scientifique, pour autant que l'autonomie et le caractère auto-administré de la science opposent une certaine résistance aux mesures de protection instaurées par l'État. À l'inverse, la trialregistration s'applique aux processus sociaux eux-mêmes, pour protéger la science contre les empiètements de l'économie. Cette procédure se distingue par le fait qu'elle intègre la dynamique propre $d u$ conflit et qu'elle protège de manière immanente l'intégrité de la science, en incitant un grand nombre d'acteurs du droit fondamental à participer au processus à partir de la logique de leurs fonctions respectives. Elle mobilise ainsi des forces susceptibles de contrecarrer les visées expansionnistes des 
réseaux pharmaceutiques. Elle fonctionne pour la science exactement comme un système immunitaire, qui identifie et combat des éléments étrangers au système de la science ${ }^{69}$. Il se trouve ici indéniablement un élément politique, qui ne fonctionne cependant pas comme un pilotage externe de la part de l'État, mais qui modifie de l'intérieur l'autoreproduction de l'activité scientifique. Des conceptions de l'obligation étatique de protection qui, au nom de la liberté de la science, imposent des contraintes de publication développées par le législateur, ne font que réduire le potentiel des processus scientifiques autonomes ${ }^{70}$. L'imposition de critères extérieurs sous-estime le besoin d'autonomie de la science et méconnaît les mécanismes de son évolution. Une telle politique doit nécessairement passer à côté des besoins sociaux réels, parce qu'elle considère les acteurs impliqués comme de simples objets de régulation. Or ceux-ci sont des (co-)auteurs responsables dans la mission de protection de l'autonomie de «leurs » domaines sociaux respectifs ${ }^{71}$. À une responsabilité globale d'organisation assignée à l'État, on oppose ainsi l'option d'un rattachement procédural des droits fondamentaux à la société elle-même. Fixer les critères du droit fondamental n'est pas une tâche exclusive de la politique étatique, mais relève avant tout de l'auto-organisation sociale. La raison déterminante en dernière instance en est que « qu'il n'existe en dehors d'un système en cours de construction pas d'information supérieure concernant les possibilités et les besoins d'ordre dans ce système ${ }^{72}$ ». Le rôle de l'État ne devrait alors pas consister à formuler des obligations globales de protection, mais devrait se limiter à mettre en place des formes indirectes de pilotage par l'organisation et la procédure ${ }^{73}$.

39 La trial-registration constitue donc une réponse adéquate à la collision des intérêts scientifiques et des intérêts économiques, parce qu'elle protège la liberté de la science par une procédure d'autorégulation de la science elle-même. À l'encontre du mécanisme proposé jusqu'à présent, fondé sur l'effet horizontal d'une diversification des sources de financement, elle offre une solution intégrant la particularité de la situation conflictuelle qui prévaut en ce domaine ${ }^{74}$. La trial-registration présente en outre une caractéristique remarquable: parce que ce sont les maisons d'éditions (structurellement liées les unes aux autres) qui organisent l'enregistrement des essais, ce mécanisme favorise la tendance à constituer un réseau spécifique d'(auto)-contrôle comme contre-pouvoir aux réseaux pharmaceutiques ${ }^{75}$. Il prend ainsi en charge le problème difficile et souvent discuté de la régulation de réseaux qui, en raison de leur structure décentralisée, ne présentent pas d'interlocuteur identifiable.

40 Toujours est-il qu'un tel réseau se compose de différents acteurs sociaux qui, pour des motifs propres à chacun, sont en mesure d'assurer la réalisation et la protection des droits fondamentaux inscrite dans le mécanisme d'enregistrement. Les revues spécialisées, qui ne peuvent se permettre de ternir leur réputation, jouent le rôle central, en faisant dépendre la publication des travaux de leur enregistrement préalable. Elles peuvent aller plus loin, et valoriser les études négatives, soit en les publiant à part, soit en instaurant une obligation de les prendre en compte lors du peerreviewing. Elles le font pour des motifs propres, désireuses qu'elles sont de préserver leur statut de médias scientifiques neutres, par opposition aux médias de masse, et de ne pas se trouver instrumentalisées comme de simples supports publicitaires ${ }^{76}$.

41 Les universités, les agences de financement de la recherche, les conseils scientifiques et les associations de médecins peuvent également contribuer de manière décisive au succès de la trial-registration ${ }^{77}$. En institutionnalisant des registres propres, des obligations internes d'enregistrement, des comités d'éthique et des procédures de 
médiation, ces acteurs renforcent l'obligation d'enregistrement instaurée par les éditeurs ${ }^{78}$. Au sein même de la pratique scientifique, l'obligation d'enregistrement renforce l'exigence de vigilance, parce qu'elle impose aux auteurs de révéler tous les détails de leur étude. Il sera de la responsabilité des médecins de s'informer par euxmêmes des résultats des recherches dans les registres et de comparer ces résultats avec les publications spécialisées. Des mécanismes globaux de contrôle pourront enfin empêcher les tentatives de contournement. Des signes de changement se font sentir quand des acteurs transnationaux comme les ONG, les médias et la public interest litigation font référence à la trial-registration dans leurs campagnes de dénonciation par le scandale ${ }^{79}$. De plus, l'OMS a fondé en 2007 le réseau International Clinical Trials Registry Platform (ICTRP) pour coordonner à l'échelle mondiale les initiatives privées et publiques en vue de l'enregistrement des études cliniques ${ }^{80}$. Les registres privés et publics, soumis à certains critères de qualité, reversent régulièrement leurs données dans un métaregistre. L'objectif est de contrôler la qualité des registres intégrés. Le métaregistre fonctionne comme un label de qualité en particulier pour les petits registres privés, en évitant les complications du projet difficilement réalisable d'un registre public unique à l'échelle mondiale.

\section{Bilan : les droits fondamentaux comme outils de règlement des conflits}

Dans cette perspective, les droits fondamentaux centrés sur et dirigés contre l'État n'apparaissent plus que comme une garantie normative spécifique dans la relation du citoyen et de l'État, et ne peuvent en aucun cas prétendre constituer un ordre de valeurs englobant l'ensemble de la société, ou ne serait-ce qu'un " cadre de référence » commun. Face au système dogmatique des droits fondamentaux centrés sur et dirigés contre l'État, il se trouve une multitude de formes de violation des droits fondamentaux qui peuvent être traitées sans faire appel à d'autres domaines de protection et d'évaluation ${ }^{81}$. Des atteintes spécifiques de droits donnent naissance à des normes spécifiques de règlement des conflits qui ne renvoient pas à des règles de priorité ou à des charges de justification d'ordre général, mais reflètent le besoin concret d'autonomie des secteurs sociaux concernés.

Comme la fonction de défense que les droits fondamentaux ont «conquise» dans la régulation des rapports entre le citoyen et l'État, le processus d'autoconstitution de la liberté de la science en conflit avec d'autres systèmes de valeurs, tel que nous venons de l'esquisser, est le résultat d'un long processus de démarcation. De la même manière que les conflits entre l'État et les citoyens ont historiquement débouché sur la conquête de droits fondamentaux centrés sur et dirigés contre l'État, la liberté de la science se constitue dans la collision avec d'autres rationalités sociales, en particulier avec la rationalité économique. La collision devient productive, dans la mesure où elle amène la science à se déterminer elle-même dans le conflit ${ }^{82}$. L'effet horizontal des droits fondamentaux n'est pas pleinement appréhendé quand on l'assimile à un simple transfert de leurs contenus défensifs objectivés, appliqués à de nouveaux contextes. Certes, les expériences historiques de droits fondamentaux centrés sur l'État s'inscrivent dans l'espace même où doit aussi se mesurer le niveau de protection de l'effet horizontal. Les obligations de protection qui incombent à l'État ne sont donc pas superflues, elles offrent au contraire une possibilité supplémentaire de réduire les 
divergences et fournissent un levier qui a sa place légitime parmi les potentiels de résolution de la collision globale entre les régimes respectifs de l'économie, de la science, du système de santées.

Dans cette relation de tension, la science a l'occasion de reformuler les limites de son autonomie sous la pression de rationalités sociales concurrentes. On peut appliquer à la naissance de droits fondamentaux transnationaux ce que Niklas Luhmann affirmait déjà du déploiement paradoxal «le plus ancien" des droits de l'homme ${ }^{84}$. C'est dans l'expérience immédiate de leur violation, dans l'épreuve d'une vive déception, que les droits fondamentaux prennent corps. C'est seulement quand le mécanisme de sélection de la publication n'est pas respecté que se concrétise sa signification pour le fonctionnement et la productivité de la science. Voilà la raison qui explique que la résolution des conflits sociaux constitue un terrain d'expérimentation pour la création $\mathrm{du}$ droit.

Les droits fondamentaux comme institution collective - la formule souligne le caractère double des droits fondamentaux, qui constituent à la fois un processus social et une norme juridique. La consolidation juridique ne doit pas menacer la dynamique sociale de protection des droits fondamentaux. C'est seulement sur cette base que ceuxci peuvent fonctionner dans leur dimension collective-institutionnelle comme des règles de conflits destinées à soutenir la différenciation sociale. Mais c'est justement pour cette raison qu'ils se dérobent aussi à l'action objectivante et uniformisante de la forme juridique. $\mathrm{Au}$ lieu de vouloir définir des standards communs des droits fondamentaux, englobant l'État et la société ${ }^{85}$, le droit devrait chercher à réagir aux " concentrations sociales de normativité86 " en s'adaptant à chaque fois au contexte particulier. Il peut modérer le déploiement créatif des dynamiques sociales, sans toutefois leur prescrire des contenus ${ }^{87}$. Ainsi comprises, les obligations juridiques de protection à l'endroit des mécanismes sociaux d'autorégulation ne visent pas des contenus, mais des procédures. Le droit a pour mission de construire des espaces protégés, permettant aux mécanismes sociaux de défense - concrètement, dans le cas présent : la trial-registration - de se développer ${ }^{88}$. En mobilisant et en multipliant les approches critiques, ce procédé permet de s'assurer que des résultats scientifiques qui ne sont pas conformes aux intérêts économiques ne seront pas manipulés. Il institutionnalise un mécanisme social qui résout le problème du biais de publication d'une manière beaucoup plus adéquate que ne pourrait le faire aucune régulation étatique. En s'orientant sur un droit qui vise à rendre possible plutôt qu'à prescrire, la trial-registration est ainsi en mesure de renforcer le régime de la science face à la dynamique expansionniste du régime économique.

\section{BIBLIOGRAPHIE}

Ahmed, I. / Sutton, A. / Riley, R. (2012) : « Assessment of Publication Bias, Selection Bias, and Unavailable Data in Meta-analyses Using Individual Participant Data: a Database Survey », BMJ, 344 ; DOI : https://doi.org/10.1136/bmj.d7762. 
Alexy, R. (2002) : «Verfassungsrecht und einfaches Recht - Verfassungsgerichtsbarkeit und Fachgerichtsbarkeit ", VVdStRL, 61, p. 7 sqq.

Applegate, W. B. / Furberg, C. D. / Byungton, R. P. (1996) : « The Multicenter Isradipine Diuretic Athersclerosis Study (Midas) », Journal of the American Medical Association, 277, p. 297-298.

Augsberg, I. (2012) : « Subjektive und objektive Dimensionen der Wissenschaftsfreiheit », in : Voigt, F. (éd.) : Freiheit der Wissenschaft. Beiträge zu ihrer Bedeutung, Normativität und Funktion, Berlin, De Gruyter, p. 65-89.

Becker, A. / Dörter, F. (2011) : «The Association between a Journal's Source of Revenue and the Drug Recommendations Made in the Articles it Publishes », Canadian Medical Association Journal, 183, p. 544-548.

Bombardier, C. / Laine, L. / Reicin, A. (2000) : « Comparison of Upper Gastrointestinal Toxicity of Rofecoxib and Naproxen in Patients with Rheumatoid Arthritis », VIGOR Study Group, The New England Journal of Medicine, 343, p. 1520-1528.

Bumke, Ch. (2009) : « Universitäten im Wettbewerb », Veröffentlichungen der Vereinigung der Deutschen Staatsrechtslehrer, 69, p. 407-461.

Chan, A. / Hróbjartsson, A. / Haahr, M. T. (2004) : « Empirical Evidence for Selective Reporting of Outcomes in Randomized Trials: Comparison of Protocols to Published Articles », Journal of the American Medical Association, 291, p. 2457-2465.

Christensen, R. / Fischer-Lescano, A. (2007) : Das Ganze des Rechts. Vom hierarchischen zum reflexiven Verständnis deutscher und europäischer Grundrechte, Berlin, Duncker \& Humblot.

Clapham, A. (2006) : Human Rights Obligations of Non-State Actors, Oxford, Oxford University Press. Conrad, D. (1965) : Freiheitsrechte und Arbeitsverfassung, Berlin, Duncker \& Humblot.

De Angelis, C. / Drazen, J. M. (2004) : « Clinical Trial Registration: a Statement from the International Committee of Medical Journal Editors », The New England Journal of Medicine, 351, p. $1250-1251$.

Dickersin, K. (1990) : « The Existence of Publication Bias and Risk Factors for its Occurrence », Journal of the American Medical Association, 263, p. 1385-1389.

Dickersin, K. / Min, Y.-I / Meinert, C. L. (1992) : « Factors Influencing Publication of Research Results. Followup of Applications Submitted to two Institutional Review Boards ", Journal of the American Medical Association, 267, p. 374-378.

Dreier, H. (1993) : Dimensionen der Grundrechte. Von der Wertordnungsjudikatur zu den objektivrechtlichen Grundrechtsgehalten, Hanovre, Hennies und Zinkeisen.

Dürig, G. (1956) : « Grundrechte und Zivilrechtsprechung », in : Maunz, Th. (éd.) : Vom Bonner Grundgesetz zur gesamtdeutschen Verfassung: Festschrift Hans Nawiasky, Munich, p. 157-210.

Dürig, G. / Maunz, Th. (éd.) (1957) : Grundgesetz. Kommentar, Munich, C. H. Beck.

Easterbrook, Ph. J. / Berlin, J. A. / Gopalan, R. / Matthews, D. R. (1991) : « Publication Bias in Clinical Research ", The Lancet, 337, p. 867-872.

Eyding, D. / Lelgemann, M. / Grouven, U. (2010) : « Reboxetine for Acute Treatment of Major Depression : Systematic Review and Meta-Analysis of Published and Unpublished Placebo and Selective Serotonin Reuptake Inhibitor Controlled Trials », BMJ, 341 ; DOI : https://doi.org/ 10.1136/bmj.c4737. 
Fanelli, D. (2010) : « Do Pressures to Publish Increase Scientists' Bias? An Empirical Support from US States Data », PLoS ONE, 5, p. 1-7.

Fischer-Lescano, A. (2008) : « Kritik der praktischen Konkordanz », Kritische Justiz, 2, p. 166-177.

Fischer-Lescano, A. / Teubner, G. (2006) : Regime-Kollisionen: Zur Fragmentierung des globalen Rechts, Francfort-sur-le-Main, Suhrkamp.

Franzen, M. (2011) : Breaking News: Wissenschaftliche Zeitschriften im Kampf um Aufmerksamkeit, Baden-Baden, Nomos.

Fugh-Berman, A. J. (2010) : The Haunting of Medical Journals: How Ghostwriting Sold "HRT" ", PLoS Medicine, 7 (9) ; DOI : https://doi.org/10.1371/journal.pmed.1000335.

Galtung, J. (1965) : «Institutionalized Conflict Resolution: A Theoretical Paradigm », Journal of Peace Research, 2, p. 348-397.

Gamillscheg, F. (1964) : « Die Grundrechte im Arbeitsrecht », Archiv für die civilistische Praxis, 164, p. 385-445.

Gardbaum, S. (2003) : « The "Horizontal Effect" of Constitutional Rights », Michigan Law Review, 102 , p. 387-459.

Gelling, L. (2013) : « Negative Results have a value », Nurse Researcher, 20, p. 3.

Gerstenberg, O. (2000) : «Privatrecht, Verfassung und die Grenzen judizieller Sozialregulierung », in : Neumann, U. / Schulz, L. (éd.) : Verantwortung in Recht und Moral, Stuttgart, Steiner, p. 141-156.

Graber, C. (1994) : Zwischen Geist und Geld: Interferenzen von Kunst und Wirtschaft aus rechtlicher Sicht, Baden-Baden, Nomos.

Han, B. (2014) : « Im digitalen Panoptikum: Wir fühlen uns frei. Aber wir sind es nicht », Der Spiegel, 2, p. 106 sq.

Hesse, K. (1999) : Grundzüge des Verfassungsrechts der Bundesrepublik Deutschland, 4 e éd., Heidelberg, C. F. Müller.

Horton, R. (1997) : « Medical Editors Trial Amnesty », The Lancet, 350, p. 756.

Institut für Qualität und Wirtschaftlichkeit im Gesundheitswesen (2011) « Bupropion, Mirtazapin und Reboxetin zur Behandlung der Depression - A05-20C-Abschlussbericht », consultable sur : https://www.iqwig.de/projekte/a05-20c.html.

Jäger, H. (1989) : Makrokriminalität: Studien zur Kriminologie kollektiver Gewalt, Francfort-sur-leMain, Suhrkamp.

Jefferson, T. / Jones, M. / Doshi, P. / Del Mar, C. B. / Heneghan, C. J. / Hama, R. / Thompson, M. J. (2012) : Neuraminidase Inhibitors for Preventing and Treating Influenza in Healthy Adults and Children ", Cochrane Database of Systematic Reviews ; DOI : 10.1002/14651858.CD008965.pub3.

Jull, A. / Chalmers, I. / Rodgers, A. (2002) : « Clinical Trials in NZ: Does Anybody Know what's Going on? », The New Zealand Medical Journal, 115, p. 269.

Karavas, V. (2006) : Digitale Grundrechte: Zur Drittwirkung der Grundrechte im Internet, Baden-Baden, Nomos.

Kesselheim, A. (2011) : « Covert Pharmaceutical Promotion in Free Medical Journals », Canadian Medical Association Journal, 183, p. 534-535.

Krimsky, Sh. (2003) : Science in the Public Interest: Has the Lure of Profits Corrupted Biomedical Research?, New York, Rowman \& Littlefield Publishers. 
Ladeur, K.-H. (2004) : Kritik der Abwägung in der Grundrechtsdogmatik, Tübingen, Mohr Siebeck.

Ladeur, K.-H. (2007) : Das Medienrecht und die Ökonomie der Aufmerksamkeit. In Sachen Dieter Bohlen, Maxim Biller, Caroline von Monaco u. a., Cologne, Halem.

Lenski, S.-Ch. (2007) : Personenbezogene Massenkommunikation als verfassungsrechtliches Problem: das Allgemeine Persönlichkeitsrecht in Konflikt mit Medien, Kunst und Wissenschaft, Berlin, Duncker \& Humblot.

Lexchin, J. / Bero, L. / Djulbegovic, B. / Clark, O. (2003) : « Pharmaceutical Industry Sponsorship and Research Outcome and Quality: Systematic Review », BMJ, 326, p. 1167-1177.

Luhmann, N. (1965) : Grundrechte als Institution: Ein Beitrag zur politischen Soziologie, Berlin, Duncker \& Humblot.

Luhmann, N. (1975) : « Die Weltgesellschaft », in : id. (éd.) : Soziologische Aufklärung, t. 2 : Aufsätze zur Theorie der Gesellschaft, Opladen, Westdeutscher Verlag, 1975, p. 51-71.

Luhmann, N. (1984) : Soziale Systeme: Grundriß einer allgemeinen Theorie, Francfort-sur-le-Main, Suhrkamp.

Luhmann, N. (1990) : Die Wissenschaft der Gesellschaft, Francfort-sur-le-Main, Suhrkamp.

Luhmann, N. (1995) : « Das Paradox der Menschenrechte und drei Formen seiner Entfaltung », in : id. (éd) : Soziologische Aufklärung, t. 6 : Die Soziologie und der Mensch, Opladen, Westdeutscher Verlag, p. 229-236.

Mathews, J. / Sweet, A. S. (2011) : « All Things in Proportion? American Rights Review and the Problem of Balancing ", Emory Law Journal, 4, p. 102-179.

Medwedjew, S. A. (1971) : Der Fall Lyssenko. Eine Wissenschaft kapituliert, Hambourg, Hoffmann \& Campe.

Nowotny, H. (2005) : " The Changing Nature of Public Science ", in : id. : (éd.) : The Public Nature of Science under Assault. Politics, Markets, Science and the Law, Berlin / Heidelberg, Springer, p. 1-28.

Nowrot, K. (2007) : « Den “Kinderschuhen” entwachsen: Die (Wieder-) Entdeckung der rechtssoziologischen Perspektive in der Dogmatik der Völkerrechtssubjektivität », Zeitschrift für Rechtssoziologie, 28, p. 21-48.

Petryna, A. (2009) : When Experiments Travel: Clinical Trials and the Global Search for Human Subjects, Princeton, Princeton University Press.

Pfeffer, C. / Olsen, B. R. (2002) : «Editorial: Journal of Negative Results in Biomedicine », Journal of Negative Results in BioMedicine, 1, p. 2.

Quack, C. / Wackerbeck, A. (2010) : « Die Verpflichtung zur Registrierung und Veröffentlichung klinischer Studien: Darstellung der europäischen Rechtslage im Vergleich zur US-Regelung nach dem FDA Amendment Act ", GesundheitsRecht, 1, p. 6-12.

Ridder, H. (1963) : Die Freiheit der Kunst nach dem Grundgesetz, Berlin, Vahlen.

Ridder, H. (1975) : Die soziale Ordnung des Grundgesetzes, Opladen, Westdeutscher Verlag.

Rüfner, W. (2011) : « Grundrechtsadressaten », in : Isensee, J. / Kirchhof, P. (éd.) : Handbuch des Staatsrechts der Bundesrepublik Deutschland, t. IX, 3e éd., Heidelberg, C. F. Müller, § 197, p. 793-842.

Ruggie, J. (2008) : « Protect, Respect, and Remedy: A Framework for Business and Human Rights », Report of the Special Representative of the Secretary-General on Human Rights and Transnational Corporations and Other Business Enterprises, A/HRC/8/5 (7 avril), p. 6-13. 
Schaaber, J. / Kochen, M. / Müller-Oerlinghausen, B. / Niebling, W. (2011) : « Warum unabhängige Arzneimittelzeitschriften und Fortbildungsveranstaltungen wichtig sind », in : Lieb, K. / Klemperer, D. / Ludwig, W.-D. / Kochen, M. (éd.) : Interessenskonflikte in der Medizin Hintergründe und Lösungsmöglichkeiten, Berlin / Heidelberg, Springer, p. 237 sqq.

Schierbeck, J. (2000) : « Operational Measures for Identifying and Implementing Human Rights Issues in Corporate Operations ", in : Eide, A. / Bergesen, O. / Goyer, P. (éd.) : Human Rights and the Oil Industry, Anvers, Intersentia, p. 161-177.

Schmidt-Aßmann, E. (2000) : « Wissenschaftsplanung im Wandel », in : Erbguth, W. / Oebbecke, J. / Rengeling, H.-W. (éd.) : Planung. Festschrift für Werner Hoppe zum 70. Geburtstag, Munich, C. H. Beck, p. 649-665.

Schmidt-Aßmann, E. (2005) : «Wissenschaft - Öffentlichkeit - Recht », in : Dreier, H. (éd.) : Rechts- und staatstheoretische Schlüsselbegriffe: Legitimität - Repräsentation - Freiheit, Symposion für Hasso Hofmann zum 70. Geburtstag, Berlin, Duncker \& Humblot, 2005, p. 67 sqq.

Schmitt, C. (1985) : « Freiheitsrechte und institutionelle Garantien der Reichsverfassung » (1931), in : id. : Verfassungsrechtliche Aufsätze aus den Jahren 1924-1954, Berlin, Duncker \& Humblot, p. 140-178.

Schott, G. / Pachl, H. / Limbach, U. (2010a) : « Finanzierung von Arzneimittelstudien durch pharmazeutische Unternehmen und die Folgen, Teil 1: Qualitative systematische Literaturübersicht zum Einfluss auf Studienergebnisse ,- protokoll und qualität », Deutsches Ärzteblatt international, 107, p. 279-285.

Schott, G. / Pachl, H. / Limbach, U. (2010b) : « Finanzierung von Arzneimittelstudien durch pharmazeutische Unternehmen und die Folgen, Teil 2: Qualitative systematische

Literaturübersicht zum Einfluss auf Autorenschaft, Zugang zu Studiendaten sowie -registrierung und Publikation », Deutsches Ärzteblatt international, 107, p. 295-301.

Schwabe, J. (1971) : Die sogenannte Drittwirkung der Grundrechte. Zur Einwirkung der Grundrechte auf den Privatrechtsverkehr, München, Goldmann.

Selznick, Ph. (1969) : Law, Society and Industrial Justice, New York, Russell Sage.

Smith, M. L. (1980) : «Publicationbias and Meta-analysis », Evaluation in Education, 4, p. 22-24.

Steinhauer, F. (2013) : « Das Grundrecht der Kunstfreiheit: Kommentar zu einem Grundlagentext von Helmut Ridder », manuscrit, Francfort-sur-le-Main.

Stern, J. / Simes, R. J. (1997) : « Publication Bias: Evidence of Delayed Publication in a Cohort Study of Clinical Research Projects », BMJ, 315, p. 640-645.

Stichweh, R. (1994) : Wissenschaft, Universität, Professionen, Francfort-sur-le-Main, Suhrkamp.

Stichweh, R. (2007) : « Einheit und Differenz im Wissenschaftssystem der Moderne », in :

Halfmann, J. / Rohbeck, J. (éd.) : Zwei Kulturen der Wissenschaft - revisited, Weilerswist, Velbrück, p. 213-228.

Stolleis, M. (2012) : Geschichte des öffentlichen Rechts in Deutschland, t. IV, Munich, C. H. Beck. Sutton, A. / Duval, S. J. / Tweedie, R. L. (2000) : « Empirical Assessment of Effect of Publication Bias on Meta-analysis », BMJ, 320, p. 1574-1577.

Teubner, G. (2006) : « Die anonyme Matrix: Zu Menschenrechtsverletzungen durch “private” transnationale Akteure », Der Staat, 45, p. 161-188. 
Teubner, G. (2012) : Verfassungsfragmente: Gesellschaftlicher Konstitutionalismus in der Globalisierung, Berlin, Suhrkamp.

Terhechte, J. P. (2011) : Konstitutionalisierung und Normativität der europäischen Grundrechte, Tübingen, Mohr Siebeck.

The PLoS Medicine Editors (Barbour, V. / Clark, J. / Jones, S.) (2011) : « Ghostwriting Revisited: New Perspectives but Few Solutions in Sight », PLoS Medicine, 8 (8), DOI : https://doi.org/10.1371/ journal.pmed.1001084.

Tonks, A. (2002) : « A Clinical Trials Register for Europe », BMJ, 325, p. 1314-1315.

Tramèr, M. R. / Reynolds, D. J. / Moore, A. /McQuay, H. (1997) : « Impact of Covert Duplicate Publication on Meta-analysis: a Case Study », BMJ, 315, p. 635-640.

Verschraegen, G. (2012) : « Differentiation and Inclusion: A Neglected Sociological Approach to Fundamental Rights ", in : Madsen, M. R. / Verschraegen, G. (éd.) : Towards a Sociology of Human Rights: New Theoretical and Empirical Contributions, Oxford, Hart.

Vesting, T. (2007) : Rechtstheorie: Ein Studienbuch, Munich, C. H. Beck.

Vesting, T. (2013) : « Die Tagesschau-App und die Notwendigkeit der Schaffung eines "Intermedienkollisionsrechts" ", in : Spiecker, I. (éd.) : Karlsruher Dialog zum Informationsrecht, Karlsruhe, KIT Scientific Publishing, p. 1 sqq.

Viellechner, L. (2013) : Transnationalisierung des Rechts, Weilerswist, Velbrück.

Viens, A. M. / Savulescu, J. (2004) : «Introduction to The Olivieri Symposium », Journal of medical ethics, 30, p. 1-7.

Wagner, E. (2011) : Der Arzt und seine Kritiker: zum Strukturwandel medizinkritischer Öffentlichkeiten am Beispiel klinischer Ethik-Kommissionen, Stuttgart, Lucius \& Lucius.

Wiethölter, R. (2003) : « Recht-Fertigungen eines Gesellschafts-Rechts », in : Joerges, C. / Teubner, G. (éd.) : Rechtsverfassungsrecht: Recht-Fertigung zwischen Privatrechtsdogmatik und Gesellschaftstheorie, Baden-Baden, Nomos, p. 13-21.

Zöllner, W. (1996) : «Regelungsspielräume im Schuldvertragsrecht: Bemerkungen zur Grundrechtsanwendung im Privatrecht und zu den sogenannten Ungleichgewichtslagen », Archiv für die civilistische Praxis, 196, p. 1-36.

\section{NOTES}

1. Le poète Diagoras de Melos adressa cette question provocatrice au prêtre qui lui montrait comme preuve de l'existence des dieux les ex-voto de marins rescapés d'un naufrage. Il fut condamné à mort pour ce mot. Cf. Marcus Tullius Cicero, De natura deorum, III, 37.

2. Eyding / Lelgemann / Grouven (2010); Institut für Qualität und Wirtschaftlichkeit im Gesundheitswesen (2011)

3. Cf. United States Court of Appeals, 7thCir. (2008), « BASF AG v. Great American Assurance Co. », 522 F. 3d 813, 816, ainsi que Krimsky (2003).

4. Fugh-Berman (2010).

5. Cf. aussi les reproches formulés dans l'affaire de l'étude Vioxx (Laboratoires Merck), où le risque d'infarctus avait été passé sous silence (Bombardier / Laine / Reicin [2000]), et dans l'affaire de l'étude sur le Tamiflu, traitement contre la grippe porcine produit par les Laboratoires Roche (Jefferson et al. [2012]). 
6. Cf. l'affaire de l'étude dite «MIDAS » sur l'efficacité des bloqueurs de calcium (fabriqués par Sandoz, qui sera plus tard intégré dans le groupe Novartis), où les chercheurs résistèrent avec succès à la manipulation. Voir à ce sujet Applegate / Furberg / Byungton (1996).

7. Ainsi par exemple le licenciement par l'Université de Toronto de la chercheuse Nancy Olivieri, qui voulait alerter le public sur les conclusions négatives de ses travaux, mais dont l'employeur recevait des subventions d'Apotex, le fabricant du médicament étudié. Voir à ce sujet Viens / Savulescu (2004).

8. Le concept apparaît déjà chez Smith (1980).

9. Chan / Hróbjartsson / Haahr (2004). Dans le domaine allemand, cf. Schott / Pachl / Limbach (2010a) et (2010b).

10. Petryna (2009).

11. Sur l'état actuel de la discussion en Allemagne cf. Rüfner (2011), Rn. [numéro dans la marge] 83-125; du point de vue historique Stolleis (2012), p. 216 sqq. ; du point de vue du droit européen, Clapham (2006) ; du point de vue du droit international, Gardbaum (2003) ; Ruggie (2008) ; du point de vue spécifique de la liberté de la recherche scientifique: Schmidt-Aßmann (2000), particulièrement p. 653.

12. Dans une perspective critique, cf. Ladeur (2004); Fischer-Lescano (2008). Pour une critique de la situation juridique aux États-Unis, Mathews / Sweet (2011), en particulier p. 116.

13. Pour la formulation du principe, cf. Hesse (1999), Rn. [numéro dans la marge] 72 ; Alexy (2002). Auparavant déjà BVerfG, BVerfGE 83,130 (Mutzenbacher).

14. Pour un point de vue critique sur la conception étatiste du devoir de sollicitude, cf. Christensen / Fischer-Lescano (2007), p. 311 sqq.

15. Pour une approche générale de cette question, cf. Dreier (1993), p. 27 sqq.

16. BVerfGE 89, 214 (Bürgschaft).

17. Cf. la vive critique de Zöllner (1996).

18. Ridder (1975), p. 90 sq.; (1963). C'est Fabian Steinhauer (2013) qui a apporté ici une clarification conceptuelle exemplaire en introduisant la notion de " collectif-institutionnel », et en démarquant ainsi le projet de Ridder de la théorie «institutionnelle » du droit fondamental développée par Carl Schmitt.

19. Schmitt (1985), p. 155.

20. La liberté de la recherche scientifique n'est pas menacée seulement par des interventions de l'État, mais aussi par des puissances sociales et en particulier économiques; elle doit donc être protégée par des mesures appropriées sur le plan du droit fondamental : c'est ce que souligne Ino Augsberg dans son article "Subjektive und objektive Dimensionen der Wissenschaftsfreiheit » (2012), p. 74.

21. Le locus classicus de la réflexion sur la violence structurelle est Galtung (1965) ; sur la macrocriminalité, Jäger (1989).

22. Ce qui ne signifie pas que la responsabilité individuelle disparaisse derrière la responsabilité collective, mais que les deux existent côte à côte, et dépendent de facteurs différents.

23. Pour un examen détaillé de ce point, cf. Teubner (2006). Steinhauer (2013) souligne lui aussi que la dimension collective-institutionnelle reste inaperçue tant qu'on n'impute ces atteintes qu'à des personnes juridiques et à des acteurs collectifs. Pour une interprétation institutionnelle de l'effet horizontal des droits fondamentaux dans l'espace transnational, cf. Viellechner (2013), p. 217 sqq.

24. Cf. l'analyse frappante de Han (2014).

25. Cette formulation dépasse la conception luhmannienne des droits fondamentaux, dans la mesure où elle ne thématise pas seulement les tendances totalisatrices de la politique, mais aussi celles d'autres systèmes partiels expansionnistes. Cf. Luhmann (1965).

26. BVerfGE 57, 295, 320 (3. Rundfunkentscheidung).

27. Vesting (2013) ; Ladeur (2007), p. 255 sqq., 268 sqq. 
28. Pour plus de précisions, cf. Teubner (2012), p. 215 sqq.

29. Pour un tableau d'ensemble, cf. Schwabe (1971), p. 12 sqq.

30. Sur l'évolution de cette doctrine, cf. US Supreme Court (1883), Civil Rights Cases, 109 US 3. Pour une position critique, cf. Gardbaum (2003).

31. Clapham (2006); Nowrot (2007).

32. Gamillscheg (1964); Conrad (1965).

33. C'est ce que souligne Gert Verschraegen (2012).

34. Stichweh (1994), p. 28.

35. Ces formules qui visent à l'origine les manipulations numériques s'appliquent également au «biais de publication» (cf. Han [2014], p. 106 sq.) On peut douter qu'il faille désigner ces pratiques par le terme de «techniques de pouvoir», comme c'est souvent le cas aujourd'hui sous l'influence de Foucault, puisque la communication ne passe pas ici par le canal du pouvoir, mais par celui de l'argent, et que les droits fondamentaux sont menacés avant même que l'argent se traduise en pouvoir.

36. Schmidt-Aßmann (2000), p. 657.

37. Ce point sera discuté plus loin, au troisième paragraphe ("Contextualisation »).

38. Sur ces tendances à une commercialisation croissante de la science, cf. Bumke (2009).

39. Medwedjew (1971).

40. Il est vrai que ce n'est pas toujours le cas. L'industrie pharmaceutique, obligée de lancer régulièrement de nouveaux produits sur le marché, cherche souvent à diriger directement les processus de production du savoir scientifique, quand elle ne pousse pas les chercheurs à falsifier ouvertement les valeurs du code scientifique.

41. D'après Niklas Luhmann, une pression externe massive sur le système scientifique conduit à une inflation du médium de la vérité. « Les promesses de vérité [...] se négocient à haut prix, sans que leur solvabilité soit suffisamment garantie. La capacité d'insertion dans le système, la vérification empirique, l'exactitude des concepts, tout cela se trouve relégué au second plan, pour ne pas contrarier l'intérêt que beaucoup attachent aux résultats de la recherche. Des phénomènes inflationnistes de ce type sont, comme la fièvre, des symptômes clairs que le système se défend contre des influences extérieures en les prenant en ligne de compte.» (Luhmann [1990], p. 623).

42. Cf. à ce sujet Luhmann (1990), p. 576 sqq. ; Stichweh (2007).

43. Sur la relation complexe entre variation, sélection et stabilisation dans l'évolution de la science, cf. Luhmann (1990), p. 583, 587 sq.

44. Sur le «droit fondamental à la communication » attaché à la liberté de la science cf., parmi de nombreuses autres références, Lenski (2007). D'autres auteurs considèrent que la fonction constitutive de l'espace public exclut l'idée d'une protection spécifique de la liberté de la science, dans un contexte général de liberté négative de publication : cf. Schmidt-Aßmann (2005).

45. Steinhauer (2013), p. 4.

46. Sur la dimension institutionnelle du droit fondamental à la liberté scientifique, cf. Cour constitutionnelle fédérale, BVerfG, BVerfGE 35, 79, 112 ; ainsi que Ino Augsberg (2012), p. 77-80.

47. Cf. à ce sujet Nowotny (2005); Lexchin et al. (2003).

48. Easterbrook et al. (1991) ; Dickersin / Min / Meinert (1992).

49. Cf. l'étude de Fanelli (2010) ; Dickersin (1990) ; Gelling (2013). Sur un «biais de décalage », cf. Stern / Simes (1997). Sur l'effet déformant des publications multiples, cf. Tramèr et al. (1997).

50. The PLoS Medicine Editors (2011).

51. Franzen (2011), p. 73 sqq., 88 sqq.

52. Cf. à ce sujet Becker / Dörter (2011) ainsi que Kesselheim (2011). Sur l'initiative des International Society of Drug Bulletins et de l'OMS visant à assurer l'indépendance des publications, cf. Schaaber et al. (2011).

53. Cf. à ce sujet Ahmed / Sutton / Riley (2012). 
54. Tonks (2002).

55. Pour une analyse de telles déductions erronées, cf. Sutton / Duval / Tweedie (2000).

56. Horton (1997).

57. Dürig (1956), p. 158 sq.

58. Sur la reformulation des droits fondamentaux dans le contexte de l'entreprise, cf. l'étude classique de Selznick (1969), p. 75 sqq., 259 sqq.; plus récemment, Schierbeck (2000), en particulier p. 168.

59. Luhmann (1965), p. 188 : la spécificité des sphères sociales mérite d'être protégée contre le nivellement induit par la politisation des rapports.

60. C'est pourtant ainsi que procède Günter Dürig, en partant de la dignité humaine comme principe supérieur de constitution du droit objectif (Dürig / Maunz [1957], Art. 1, Rn. [numéro dans la marge] 5 sqq.

61. Telle est du reste la pratique du Tribunal constitutionnel fédéral, qui fonde la description juridique de la science, de l'art et d'autres domaines partiels sur la compréhension qu'ils ont d'eux-mêmes : cf. BVerfGE 111, 333, 354 ; ordonnance du 20.07.2010 - 1 BvR 748/06 -, JZ 2011, p. 308-313, 308. Pour une étude détaillée du sujet, cf. Augsberg (2012), p. 74 sq., 84.

62. Thomas Vesting parle à ce propos de "conventions sociales et [de] savoir implicite». Cf. Vesting (2007), p. 95 sqq.

63. On peut recourir ici au concept, développé par Wiethölter, de la procéduralisation comme libération de normativité sociale. Cf. Wiethölter (2003), en particulier p. 18 sqq.

64. Les États-Unis en ont fourni le modèle avec le «FDA Amendments Act » de 2007, Food and Drug Administration: FDA Amendments Act (FDAAA) of 2007, public law no. 110-85 § 801, consultable en ligne à l'adresse suivante: http://www.gpo.gov/fdsys/pkg/PLAW-110publ85/pdf/ PLAW-110publ85.pdf. Sur la situation juridique en Europe, cf. Quack / Wackerbeck (2010).

65. Des premiers jalons avaient été posés avec le registre officiel américain ClinicalTrials.gov ou bien le Deutsche Register Klinischer Studien à la clinique universitaire de Fribourg (www.germanctr.de). La brûlante actualité du thème a depuis suscité la création, au niveau européen, non seulement de la banque de données EudraCT, à laquelle seules les autorités des États-membres ont accès, mais aussi de banques de données publiques telles que EudraPharm et le Clinicaltrialregister (www.clinicaltrialsregister.eu).

66. De Angelis / Drazen (2004). Il existe également quelques revues en ligne qui publient principalement des études négatives, comme par exemple le Journal of Negative Results in Biomedicine (http://www.jnrbm.com/). Cf. à ce sujet Pfeffer / Olsen (2002).

67. De la même manière, les revues peuvent lutter contre le recours aux prête-plumes en conditionnant la publication à la description détaillée du rôle de chaque participant et des sources de financement.

68. Cette différence décisive est signalée par Han (2014). La protection des droits fondamentaux contre la «matrice anonyme » doit donc être conçue autrement que celle qui vise à prévenir les atteintes de l'État. Cf. à ce sujet Teubner (2006), p. 181 sqq.

69. «Les systèmes sociaux ont besoin de contradictions pour entretenir leur système immunitaire, pour assurer leur autoreproduction dans des circonstances difficiles. » Luhmann (1984), p. 526 ; (1990), p. 623.

70. C'est l'un des problèmes que comporte la nouvelle directive européenne sur les études cliniques (consultable à l'adresse suivante: http://register.consilium.europa.eu/doc/srv? $\mathrm{l}=\mathrm{EN} \& \mathrm{t}=\mathrm{PDF} \& \mathrm{gc}=$ true\&sc=false\&f=ST\%2017866\%202013\%20INIT), adoptée par le Conseil des ministres européen le 20.12.2013. Dans la mesure où cette directive évoque une obligation d'enregistrement pour les études cliniques, elle réagit à la pression sociale émanant des associations de médecins, des comités d'éthique, de nombreuses ONG et des États-membres, visant à maintenir le niveau de protection de la trial-registration sociale. Un premier projet de directive datant de juillet 2011 était encore exclusivement orienté vers la libéralisation et la 
stabilisation du marché pharmaceutique européen. Mais quand la directive, au-delà de la simple obligation d'enregistrement, définit de surcroît les normes et le format concret d'établissement des clinical study reports, elle sape la diversité des formes que peut prendre la publication des résultats scientifiques, avec des effets imprévisibles sur l'activité de recherche.

71. Gerstenberg (2000) ; Karavas (2006), p. 87 sqq., 99.

72. Cf. Stichweh (1994), p. 84.

73. Cf. Augsberg (2012), p. 80.

74. Sur la diversification des sources de financement, cf. Graber (1994), p. 227 sqq.

75. Elke Wagner décrit une évolution similaire dans son livre (2011).

76. Sur certaines initiatives prises dans le monde de l'édition scientifique, cf. par exemple Franzen (2011), p. 246 sqq.

77. La déclaration d'Helsinki de l'Association médicale mondiale, à la suite des élargissements de 2000 et 2008, énonce ainsi l'obligation «d'enregistrer toute étude clinique [...] avant même de recruter le premier sujet d'expérience, dans une banque de données publiquement accessible » (art. 35) et de publier les études négatives (art. 36). Document consultable à l'adresse suivante : https://www.wma.net/policies-post/wma-declaration-of-helsinki-ethical-principles-for-

medical-research-involving-human-subjects/.

78. C'est par exemple le cas du comité d'éthique de la Faculté de Médecine de Fribourg (https:// www.uniklinik-freiburg.de/ethikkommission/antragstellung/gemaess-mpg.html). Sur ce sujet en général, cf. Jull / Chalmers / Rodgers (2002).

79. Sur le rôle des médias et la stratégie du scandale dans la mise en évidence du «biais de publication ", cf. les exemples donnés ci-dessus.

80. World Health Organization, International Clinical Trials Registry Platform (ICTRP), 2012, consultable à l'adresse suivante : https://www.who.int/clinical-trials-registry-platform.

81. Cf. Luhmann (1965), p. 36.

82. Pour une analyse de l'autonomie de la science, cf. Augsberg (2012), p. 74 sqq.

83. Sur la notion de « régime », cf. Fischer-Lescano / Teubner (2006).

84. Luhmann (1995), p. 222.

85. À l'instar par exemple des auteurs qui interprètent les droits fondamentaux européens comme l'expression d'une conviction commune. Cf. Terhechte (2011), p. 3 sqq.

86. Sur cette idée, cf. Selznick (1969), p. 32 sqq. ; Luhmann (1965), p. 192.

87. Sur le primat structurel de l'adaptation et de l'apprentissage dans la société mondiale, et sur la nécessité pour le droit d'admettre les attentes cognitives, cf. Luhmann (1975).

88. Sur un tel droit impartialement partial, qui prend parti pour l'autonomie sociale, mais articule d'une manière impartiale ce parti-pris, cf. Wiethölter (2003). Sur la fonction de modération du droit, cf. aussi Christensen /Fischer-Lescano (2007), p. 316.

\section{INDEX}

Mots-clés : droits fondamentaux, biais de publication, effet horizontal

Schlüsselwörter : Grundrechte, Publication Bias, Drittwirkung 


\section{AUTEURS}

\section{ISABELL HENSEL}

Isabell Hensel est chercheuse à l'Université Viadrina à Francfort-sur-Oder. Pour plus d'informations, voir la notice suivante.

\section{GUNTHER TEUBNER}

Gunther Teubner est professeur émérite de droit privé et de sociologie du droit à l'Université de Francfort-sur-le-Main. Pour plus d'informations, voir la notice suivante. 